\title{
Vibration of composite beams with two overlapping delaminations
}

\begin{abstract}
Delaminations in composite laminates may develop from small cracks due to fabrication and impact loading, or from places of high stress concentration. The locations of the delaminations are not determinate. In this research, an analytical solution for the free vibration of a composite beam with two overlapping delaminations is presented. The delaminated beam is analyzed as seven interconnected beams using the delaminations as their boundaries. The continuity and equilibrium conditions are satisfied between the adjoining regions of the beams. Classical beam theory is applied to each of the beams. Complex vibration behaviors emerge for different sizes and locations of the delaminations. Comparison with analytical results reported in the literature verifies the validity of the present solution.
\end{abstract}

Keywords Vibration - Delaminations - Composite beams $\cdot$ Natural frequency $\cdot$ Mode shape

\section{Introduction}

Delamination is probably the most frequently occurring damage in composite laminates due to its weak interlaminar strength. Delaminations may arise as a result of either imperfect fabrication processes or impact during service. The presence of the delaminations is known to cause strength and stiffness degradation, as well as changes of the vibration characteristics of the laminates. In particular, delaminations reduce the natural frequency, which may cause resonance if

Christian N Della wishes to thank Nangyang Technological University for the scholarship.

D. Shu $(\bowtie) \cdot$ C. N. Della

School of Mechanical and Production Engineering, Nanyang Technological University, Singapore 639798

E-mail: mdshu@ntu.edu.sg

Tel.: +65-67904440; Fax: +65-67911859

Y. Zhao

State Key Laboratory of Nonlinear Mechanics, Institute of Mechanics, Chinese Academy of Sciences, Beijing 100080, China

E-mail: yzhao@lnm.imech.a.c.cn the reduced frequency is close to the working frequency. It is imperative that we should be able to predict the changes in the frequency, as well as the mode shape, in a dynamic environment.

To study the free vibration of an isotropic beam with a through-width delamination, Wang et al. [1] presented an analytical solution by treating the delaminated beam as four Euler-Bernoulli beams that are joined together. By applying appropriate boundary and continuity conditions, the response of the beam was obtained as a whole. However, the vibration modes are physically inadmissible for off-midplane delaminations because the delaminated layers were assumed to deform 'freely' without touching each other and thus have different transverse deformations ('free mode'). Mujumdar and Suryanarayan [2] then proposed a model based on the assumption that the delaminated layers are 'constrained' to have identical transverse deformations ('constrained mode'). This 'constrained mode' approach was extended by Shu and Fan [3] on a bimaterial beam and $\mathrm{Hu}$ and $\mathrm{Hwu}$ [4] on a sandwich beam to include the effects of the rotary inertia and transverse shear deformation. A similar 'constrained mode' approach was proposed by Tracy and Pardoen [5] on a composite beam. Valoor and Chandrashekhara [6] extended this model for thick composites to include the effects of the transverse shear deformation and the rotary inertia. In addition, the Poisson effect was included due to its significance in the analysis of angle-ply laminated beams. However, the "constrained mode' analysis failed to predict the opening in the mode shapes found in the experiments by Shen and Grady [7]. To simulate the 'open' and 'closed' behavior between the delaminated surfaces, Luo and Hanagud [8] presented an analytical model based on the Timoshenko beam theory, which uses piecewise-linear springs. The spring stiffness is then set to be equal to zero (0) for the 'free mode' and infinity $(\infty)$ for the 'constrained mode'. Saravanos and Hopkins [9] developed an analytical solution for predicting natural frequencies, mode shapes and modal damping of a delaminated composite beam based on a general laminate theory which involves kinematic assumptions representing the discontinuities in the in-plane and through-the-thickness 


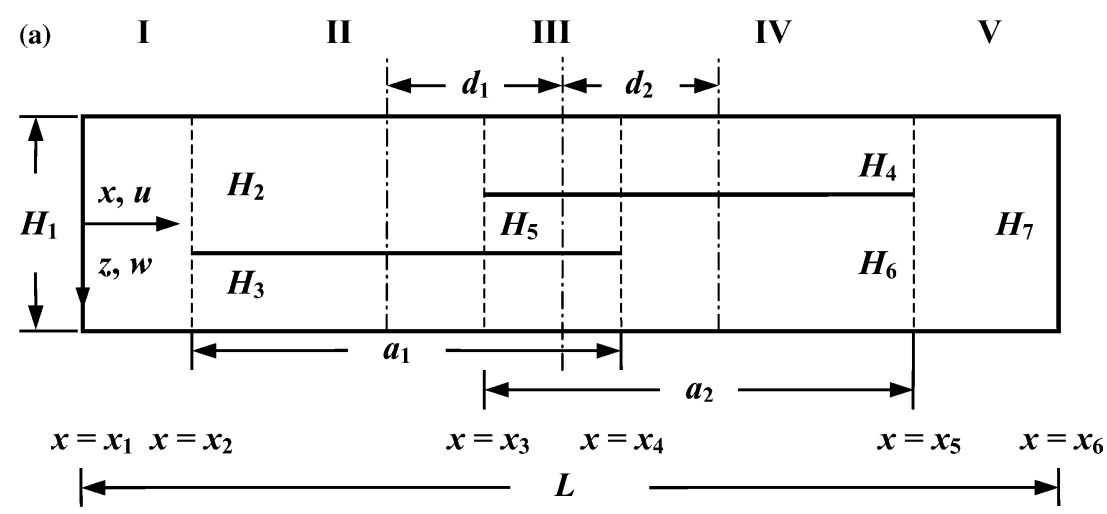

(b)

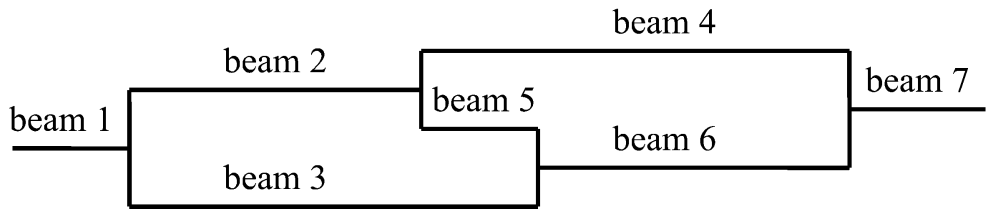

Fig. 1 (a) A model of a beam with two overlapping delaminations; (b) The delaminated beam is modeled by seven interconnected beams

displacements across each delamination crack. Chakraborty et al. [10] presented a finite element method to study the free vibration of delaminated asymmetric composite beams using refined locking free first-order shear deformable elements.

The above studies are on one-dimensional beam-plates with a single delamination. Two-dimensional plates with a single delamination have been numerically investigated. Zak et al. $[11,12]$ presented a finite element method using the first-order shear deformation theory. They modeled the delaminated region by using additional boundary conditions at the delamination fronts. Finite element methods using the higher-order deformation theory were presented by Chattopadhyay et al. [13], Radu and Chattopadhyay [14] and $\mathrm{Hu}$ et al. [15].

The multiple delaminations have been studied by a number of researchers. Shu [16] presented an analytical solution to study a sandwich beam with double delaminations. His study emphasized on the influence of the contact mode, 'free' and 'constrained', between the delaminated layers and the local deformations at the delamination fronts. Lestari and Hanagud [17] studied a composite beam with multiple delaminations using the Euler-Bernoulli beam theory with piecewise-linear springs to simulate the 'open' and 'closed' behavior between the delaminated surfaces. Lee et al. [18] studied a composite beam with arbitrary lateral and longitudinal multiple delaminations using the 'free mode' analysis and assumed a constant curvature at the multiple-delamination tip. Shu and Della $[19,20]$ used the 'free mode' and 'constrained mode' analyses to study a composite beam with two overlapping delaminations and two non-overlapping delaminations. Their study emphasized on the influence of a second short delamination on the natural frequency and mode shape of the delaminated beam. Finite element analyses were presented by Ju et al. [21] using the Timoshenko beam theory and Lee [22] using the layerwise theory.
Similar to the single delamination case, two-dimensional plates with multiple delaminations have been numerically investigated. Finite element methods were developed by Ju et al. [23] using the Mindlin plate theory, Cho and Kim [24] using the higher-order zig-zag theory and Kim et al. [25,26] using the layerwise theory.

In this research, an analytical solution for the free vibration of composite beams with two overlapping delaminations is presented. The delaminated beam is analyzed as seven interconnected Euler-Bernoulli beams using the delaminations as their boundaries. The continuity and equilibrium conditions are satisfied between adjoining regions of the beams. Both the 'free mode' and 'constrained mode' analyses in the delamination vibration are used. The influence of the differential-stretching is included in the analysis. Results show that the sizes and locations of the delaminations significantly influence the frequency and the mode shape of the beam. The results of the present solution agree well with the analytical results presented in the literature.

\section{Formulation}

In this section the analytical solution for the vibration of beams with two overlapping delamination is formulated. Figure 1 (a) shows a beam with length $L$ and thickness $H_{1}$ with two overlapping delaminations. The upper and lower delaminations are of lengths $a_{1}$ and $a_{2}$, respectively, and located at distances $d_{1}$ and $d_{2}$ away from the center of the beam, respectively. The beam is considered as seven beams connected at the delamination boundaries (Fig.1 (b)). The two delaminations divide the beam into three layers with thickness $\mathrm{H}_{3}, \mathrm{H}_{4}$ and $\mathrm{H}_{5}$.

Two assumptions were made in the literature on delamination buckling and vibrations. The first concerns the complicated changing contact between the delaminated layers. 


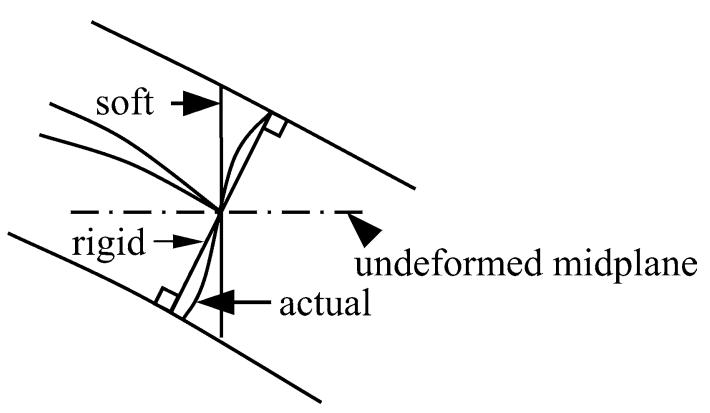

Fig. 2 Illustration of the 'rigid' and 'soft' connectors on the deformed beam with an exaggerated deformation

Wang et al. [1] assumed that the delaminated layers deformed 'freely' without touching each other, which was shown to be physically inadmissible [2]. Mujumdar and Suryanarayan [2] then proposed a 'constrained' mode where the delaminated layers are assumed to be in touch along their whole length all the time, but are allowed to slide over each other. The second assumption concerns the deformation of the delamination fronts. Two possibilities were examined by Shu and Mai $[27,28,29]$, that is, the 'rigid' and the 'soft' connectors. The cross-section in a 'rigid connector' remains perpendicular to the deformed midplane of the beam, and thus the differential stretching between the delaminated beams is taken account of. The cross-section of a 'soft connector' remains perpendicular to the undeformed beam, and thus the differential stretching is neglected. Studies by Shu and Mai [28] show that the real delamination fronts lie between the two connectors but are closer to the 'rigid connector' (Fig. 2). In this research, the 'free' and 'constrained' modes and the 'rigid connector' are considered in the analysis of the composite beam.

\section{1 'Free mode'}

For the 'free mode', the governing equations for the free vibration of a delaminated beam are

$E I_{i} \frac{\partial^{4} w_{i}}{\partial x^{4}}+\rho_{i} A_{i} \frac{\partial^{2} w_{i}}{\partial t^{2}}=0 \quad(i=1-7)$

where $E I_{i}$ is the bending stiffness of the $i$-th beam, $\rho_{i}$ is the mass density and $A_{i}$ is the cross sectional area. For planestress problems, $E$ is the Young's modulus. For plane strain problems, $E$ should be replaced by an equivalent Young's modulus $E=E /\left(1-v^{2}\right)$, where $v$ is the Poisson's ratio.

For free vibrations

$w_{i}(x, t)=W_{i}(x) \sin (\omega t)$

where $\omega$ is the natural frequency and $W_{i}(x)$ is the mode shape. Substituting Eq. (2) in Eq. (1), one can obtain the generalized solutions of the differential equation in Eq. (1) as

$$
\begin{aligned}
W_{i}(x)= & C_{i} \cos \left(\lambda_{i} \frac{x}{L}\right)+S_{i} \sin \left(\lambda_{i} \frac{x}{L}\right) \\
& +C H_{i} \cosh \left(\lambda_{i} \frac{x}{L}\right)+S H_{i} \sinh \left(\lambda_{i} \frac{x}{L}\right)
\end{aligned}
$$

where

$$
\lambda_{i}^{4}=\frac{\omega^{2} \rho_{i} A_{i}}{E I_{i}} L^{4}
$$

and where $\lambda_{i}$ is the non-dimensional frequency. By applying the boundary conditions and continuity conditions to the beams, the 28 unknown coefficients $C_{i}, S_{i}, C_{i}$ and $S H_{i}$ $i=1-7)$ can be determined.

The appropriate boundary conditions that can be applied at the supports, $x=x_{1}$ and $x=x_{4}$, are $W_{i}=0$ and $W_{i}^{\prime}=0$, if the end of the beam is clamped; $W_{i}=0$ and $W_{i}^{\prime \prime}=0$, if it is simply supported; $W_{i}^{\prime \prime}=0$ and $W_{i}^{\prime \prime \prime}=0$, if it is free, where $i=1$ to 7 and prime / denotes differentiation with respect to the $x$-coordinate.

The continuity conditions for deflection and slope at the delamination boundary $x=x_{2}$ are

$W_{1}=W_{2}$

$W_{1}=W_{3}$

$W_{1}^{\prime}=W_{2}^{\prime}$

$W_{1}^{\prime}=W_{3}^{\prime}$

From Fig.3, the continuity for shear force and bending moment at the delamination boundary $x=x_{2}$ are

$V_{1}=V_{2}+V_{3}$

$M_{1}=M_{2}+M_{3}+P_{3}\left(\frac{H_{1}}{2}-\frac{H_{3}}{2}\right)-P_{2}\left(\frac{H_{1}}{2}-\frac{H_{2}}{2}\right)$

where

$V_{i}=-E I_{i} W_{i}^{\prime \prime \prime}$

$M_{i}=-E I_{i} W_{i}^{\prime \prime} \quad(i=1-3)$

The third and fourth terms on the right side of Eq.(10) represent the contribution to the bending moment from the differential stretching between beam 2 and beam 3 for a 'rigid connector', which is neglected for a 'soft connector' [28]. Each of the delamination boundaries $x=x_{3}, x=x_{4}$ and $x=x_{5}$ provides 6 equations and a total of 24 equations can be set up for all the delamination boundaries.

The five axial forces $P_{2}, P_{3}, P_{4}, P_{5}$ and $P_{6}$ that appear in the moment continuity condition are still unknown, so five additional equations are needed. At $x=x_{2}$ and $x=x_{5}$, from the axial force balance, we have

$P_{1}=P_{2}+P_{3}=0 ; P_{3}=-P_{2}$

$P_{7}=P_{4}+P_{6}=0 ; P_{4}=-P_{6}$

At $x=x_{3}$ and $x=x_{4}$, from the axial force balance, we have
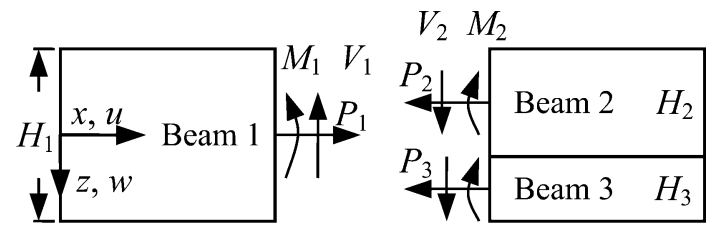

$V_{3} M_{3}$

Fig. 3 Continuity of shear force and bending moment at the delamination boundary $x=x_{2}$ 


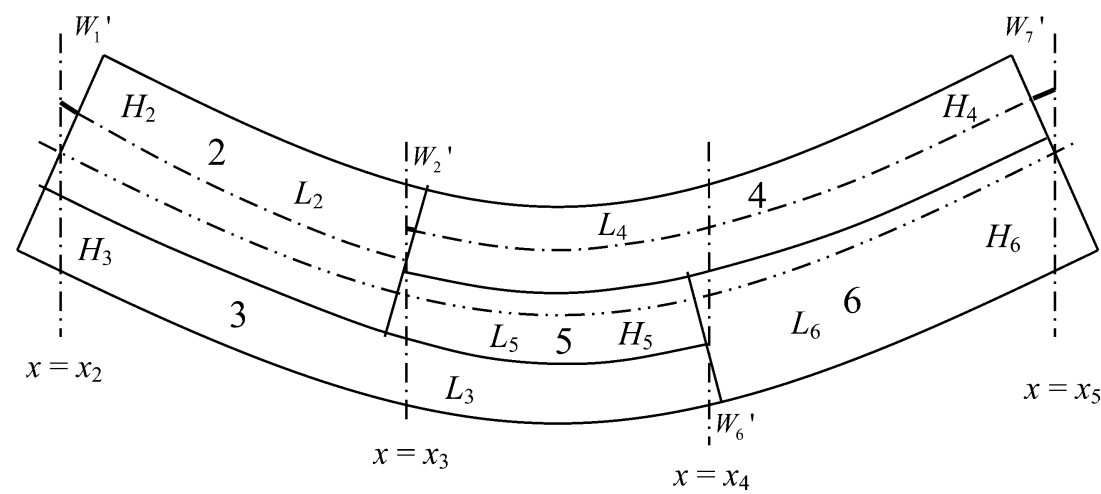

Fig. 4 The vibration configuration between $x=x_{2}$ and $x=x_{5}$. The lengths of the central lines remain unchanged

$$
\begin{aligned}
& P_{2}=P_{4}+P_{5} \\
& P_{6}=P_{3}+P_{5}
\end{aligned}
$$

If the beam does not vibrate, $P_{2}, P_{3}, P_{4}, P_{5}$ and $P_{6}$ are all zero, because they are defined as the axial forces present during the vibration of the beam. The axial length of the beam between $x=x_{2}$ and $x=x_{5}$ remains equal before and during vibration (Fig. 4). This leads to the following equations.

$$
\begin{aligned}
& \left(\frac{H_{1}}{2}-\frac{H_{2}}{2}\right) W_{1}^{\prime}\left(x_{2}\right)+L_{2}+\left(\frac{H_{2}}{2}-\frac{H_{4}}{2}\right) W_{2}^{\prime}\left(x_{3}\right) \\
& +L_{4}-\left(\frac{H_{7}}{2}-\frac{H_{4}}{2}\right) W_{7}^{\prime}\left(x_{5}\right)=L_{2 o}+L_{4 o} \\
& \left(\frac{H_{1}}{2}-\frac{H_{2}}{2}\right) W_{1}^{\prime}\left(x_{2}\right)+L_{2}+\left(\frac{H_{5}}{2}-\frac{H_{2}}{2}\right) W_{2}^{\prime}\left(x_{3}\right) \\
& \quad+L_{5}-\left(\frac{H_{6}}{2}-\frac{H_{5}}{2}\right) W_{6}^{\prime}\left(x_{4}\right) \\
& \quad+L_{6}-\left(\frac{H_{6}}{2}-\frac{H_{7}}{2}\right) W_{7}^{\prime}\left(x_{5}\right)=L_{2 o}+L_{5 o}+L_{6 o} \\
& \left(\frac{H_{3}}{2}-\frac{H_{1}}{2}\right) W_{1}^{\prime}\left(x_{2}\right)+L_{3}-\left(\frac{H_{3}}{2}-\frac{H_{6}}{2}\right) W_{6}^{\prime}\left(x_{4}\right) \\
& +L_{6}-\left(\frac{H_{6}}{2}-\frac{H_{7}}{2}\right) W_{7}^{\prime}\left(x_{5}\right)=L_{3 o}+L_{6 o}
\end{aligned}
$$

where $L_{i o}$ represents the length of the $i$ th beam segment before vibration, $H_{i}$ is the thickness of the $i$ th beam segment. If $L_{i}$ represents the length of the $i$ th beam segment,

$$
L_{i}-L_{i o}=\frac{P_{i} L_{i o}}{E A_{i}} \quad(i=2-6)
$$

Substituting Eq. (20) into Eqs. (17) through (19) leads to

$$
\begin{aligned}
\frac{P_{2} L_{2 o}}{E A_{2}} & +\frac{P_{4} L_{4 o}}{E A_{4}} \\
= & -\left(\frac{H_{1}}{2}-\frac{H_{2}}{2}\right) W_{1}^{\prime}\left(x_{2}\right)-\left(\frac{H_{2}}{2}-\frac{H_{4}}{2}\right) W_{2}^{\prime}\left(x_{3}\right) \\
& +\left(\frac{H_{7}}{2}-\frac{H_{4}}{2}\right) W_{7}^{\prime}\left(x_{5}\right)
\end{aligned}
$$

$$
\begin{aligned}
& \frac{P_{2} L_{2 o}}{E A_{2}}+\frac{P_{5} L_{5 o}}{E A_{5}}+\frac{P_{6} L_{6 o}}{E A_{6}} \\
& =-\left(\frac{H_{1}}{2}-\frac{H_{2}}{2}\right) W_{1}^{\prime}\left(x_{2}\right)-\left(\frac{H_{5}}{2}-\frac{H_{2}}{2}\right) W_{2}^{\prime}\left(x_{3}\right) \\
& \quad+\left(\frac{H_{6}}{2}-\frac{H_{5}}{2}\right) W_{6}^{\prime}\left(x_{4}\right)+\left(\frac{H_{6}}{2}-\frac{H_{7}}{2}\right) W_{7}^{\prime}\left(x_{5}\right) \\
& \frac{P_{3} L_{3 o}}{E A_{3}}+\frac{P_{6} L_{6 o}}{E A_{6}} \\
& =-\left(\frac{H_{3}}{2}-\frac{H_{1}}{2}\right) W_{1}^{\prime}\left(x_{2}\right)+\left(\frac{H_{3}}{2}-\frac{H_{6}}{2}\right) W_{6}^{\prime}\left(x_{4}\right) \\
& \quad+\left(\frac{H_{6}}{2}-\frac{H_{7}}{2}\right) W_{7}^{\prime}\left(x_{5}\right)
\end{aligned}
$$

Thus we have the number of equations to solve the simultaneous equations.

\section{2 'Constrained mode'}

The delaminated beam is analyzed as five beam segments I-V (Fig. 1). The governing equations for beam segments I-V are

$$
\begin{aligned}
& E I_{i} \frac{\partial^{4} w_{i}}{\partial x^{4}}+\rho_{i} A_{i} \frac{\partial^{2} w_{i}}{\partial t^{2}}=0 \quad(i=I \text { and } V) \\
& \left(E I_{2}+E I_{3}\right) \frac{\partial^{4} w_{I I}}{\partial x^{4}}+\left(\rho_{2} A_{2}+\rho_{3} A_{3}\right) \frac{\partial^{2} w_{I I}}{\partial t^{2}}=0 \\
& \left(E I_{3}+E I_{4}+E I_{5}\right) \frac{\partial^{4} w_{I I I}}{\partial x^{4}} \\
& \quad+\left(\rho_{3} A_{3}+\rho_{4} A_{4}+\rho_{5} A_{5}\right) \frac{\partial^{2} w_{I I I}}{\partial t^{2}}=0 \\
& \left(E I_{4}+E I_{6}\right) \frac{\partial^{4} w_{I V}}{\partial x^{4}}+\left(\rho_{4} A_{4}+\rho_{6} A_{6}\right) \frac{\partial^{2} w_{I V}}{\partial t^{2}}=0
\end{aligned}
$$

The generalized solutions for the 'constrained mode' are identical in form to the 'free mode'. The unknown coefficients $C_{i}, S_{i}, C H_{i}$ and $S H_{i}(i=\mathrm{I}-\mathrm{V})$, however, are reduced to 20 coefficients, which can be determined from the 4 boundary conditions and 16 continuity conditions.

The boundary conditions for the 'constrained mode' are identical to the boundary conditions of the 'free mode'. The continuity conditions for deflection, slope, shear force and bending moment at $x=x_{2}$ are, 


$$
\begin{aligned}
& W_{I}=W_{I I} \\
& W_{I}^{\prime}=W_{I I}^{\prime} \\
& E I_{1} W_{I}^{\prime \prime \prime}=\left(E I_{2}+E I_{3}\right) W_{I I}^{\prime \prime \prime} \\
& E I_{1} W_{I}^{\prime \prime}+P_{3}\left(\frac{H_{1}}{2}-\frac{H_{3}}{2}\right)-P_{2}\left(\frac{H_{1}}{2}-\frac{H_{2}}{2}\right) \\
& \quad=\left(E I_{2}+E I_{3}\right) W_{I I}^{\prime \prime}
\end{aligned}
$$

Similarly, we can derive the continuity conditions at $x=$ $x_{3}, x=x_{4}$ and $x=x_{5}$. For the 'constrained mode', the boundary conditions and continuity conditions provide 20 homogeneous equations for 20 unknown coefficients $C_{i}, S_{i}$, $\mathrm{CH}_{i}$ and $\mathrm{SH}_{i}$.

\section{Results and discussion}

This section presents the results obtained using the analytical model described above to study a homogeneous clampedclamped beam with two overlapping delaminations. To verify the accuracy of the present results, a comparison with published results on a homogeneous beam with a single delamination is made. The first three non-dimensional natural frequencies of a clamped-clamped beam with a midplane and central delamination having various lengths are compared with the analytical results of Wang et al. [1] and FEM results of Lee [22]. Tables 1-3 show good agreement between the present results and the analytical and FEM results.

Figure 5 shows the influence of the overlapping length $a_{o}$ and $a_{t}$ on the frequency of the beam, where $a_{t}$ is the distance between the opposite ends of the two delaminations along the beam length (from the left end of the lower delamination to the right end of the surface delamination). The fundamental frequency, $\omega$, is normalized with respect to the frequency of an undelaminated beam, $\omega_{o}$. The lengths of the two delaminations are equal, $a_{1}=a_{2}$, and $a_{t}+a_{o}=a_{1}+a_{2}$. The spanwise locations of the delaminations are determined by $d_{1}=d_{2}=\left(a_{1}-a_{o} a_{t}\right) / 2$. The surface delamination is at $H_{4}=0.2 H_{1}$ and the other delamination is at midplane $\left(H_{3}=0.5 H_{1}\right)$. The overlap $a_{o}$ lies symmetrically about the center of the beam. $a_{o}=0.0$ represents two non-overlapping delaminations, where the left end of the surface delamination and the right end of the midplane delamination are along the midspan of the beam. Since the formulation is for two overlapping delaminations, the frequency is approximated by having $d_{1}=d_{2}=a_{1} / 2-5 \times 10^{-5}$ L. $a_{o}=a_{t}$ represents two fully overlapping delaminations and the frequency is approximated by having $d_{1}=d_{2}=5 \times 10^{-5} L$. For the 'constrained mode', $\omega / \omega_{o}$ decreases rapidly after a threshold value of about $a_{t} / L=0.4$. The presence of an overlap, $a_{o}$, further decreases the frequency. However, the frequencies for $a_{o} / a_{t}=0.2,0.4$, 0.6 are close to one another. This indicates that the influences of two overlapping delaminations with $a_{o} / a_{t}=0.2,0.4,0.6$ are almost the same. Similarly, for the 'free mode', $\omega / \omega_{o}$ decreases rapidly after a threshold value of about $a_{t} / L=0.4$ and further decreases with increasing $a_{o}$. However, a significant decrease in the frequency is observed as $a_{o}$ increases.
Table 1 Non-dimensional primary frequency $\left(\lambda^{2}\right)$ of a clampedclamped isotropic beam with a midplane delamination

\begin{tabular}{llll}
\hline $\begin{array}{l}\text { Delamination } \\
\text { length, } a / L\end{array}$ & $\begin{array}{l}\text { Present } \\
\text { Cons and Free }\end{array}$ & Analytical [1] & FEM [22] \\
\hline 0.00 & 22.37 & 22.39 & 22.36 \\
0.10 & 22.37 & 22.37 & 22.36 \\
0.20 & 22.36 & 22.35 & 22.35 \\
0.30 & 22.24 & 22.23 & 22.23 \\
0.40 & 21.83 & 21.83 & 21.82 \\
0.50 & 20.89 & 20.88 & 20.88 \\
0.60 & 19.30 & 19.29 & 19.28 \\
0.70 & 17.23 & 17.23 & 17.22 \\
0.80 & 15.05 & 15.05 & 15.05 \\
0.90 & 13.00 & 13.00 & 12.99 \\
\hline
\end{tabular}

Table 2 Non-dimensional secondary frequency $\left(\lambda^{2}\right)$ of a clampedclamped isotropic beam with a midplane delamination

\begin{tabular}{llll}
\hline $\begin{array}{l}\text { Delamination } \\
\text { length, } a / L\end{array}$ & $\begin{array}{l}\text { Present } \\
\text { Cons and Free }\end{array}$ & Analytical [1] & FEM [22] \\
\hline 0.00 & 61.67 & 61.67 & 61.61 \\
0.10 & 60.81 & 60.76 & 60.74 \\
0.20 & 56.00 & 55.97 & 55.95 \\
0.30 & 49.00 & 49.00 & 48.97 \\
0.40 & 43.89 & 43.87 & 43.86 \\
0.50 & 41.52 & 41.45 & 41.50 \\
0.60 & 41.04 & 40.93 & 41.01 \\
0.70 & 40.82 & 40.72 & 40.80 \\
0.80 & 39.07 & 39.01 & 39.04 \\
0.90 & 35.39 & 35.38 & 35.38 \\
\hline
\end{tabular}

Table 3 Non-dimensional third frequency $\left(\lambda^{2}\right)$ of a clamped-clamped isotropic beam with a midplane delamination

\begin{tabular}{llll}
\hline $\begin{array}{l}\text { Delamination } \\
\text { length, } a / L\end{array}$ & $\begin{array}{l}\text { Present } \\
\text { Cons and Free }\end{array}$ & Analytical [1] & FEM [22] \\
\hline 0.00 & 120.90 & 120.91 & 120.68 \\
0.10 & 120.83 & 120.81 & 120.62 \\
0.20 & 118.87 & 118.76 & 118.69 \\
0.30 & 109.16 & 109.04 & 109.03 \\
0.40 & 93.59 & 93.57 & 93.51 \\
0.50 & 82.29 & 82.29 & 82.23 \\
0.60 & 77.69 & 77.64 & 77.64 \\
0.70 & 77.18 & 77.05 & 77.12 \\
0.80 & 75.43 & 75.33 & 75.39 \\
0.90 & 69.19 & 69.17 & 69.16 \\
\hline
\end{tabular}

The difference between the 'constrained mode' and the 'free mode' frequencies increases as $a_{t}$ increases (Fig. 5). The difference between the two frequencies further increases as $a_{o}$ increases. This can be explained by the opening in the 'free mode' mode shapes. The 'free mode' mode shapes for $a_{t} / L=$ $0.3,0.5,0.8$ at $a_{o} / a_{t}=0.6$ are computed and shown in Fig. 6 . Three types of vibration modes are observed, local, mixed and global vibration modes. In a global vibration mode, the amplitudes of beams 2-6 are almost equal (Fig. 6(a)), in a mixed vibration mode, the amplitudes of beams 2-6 are comparable (Fig. 6(b)), and in a local vibration mode, beam 4 will have much higher amplitude than beams 2, 3, 5 and 6 (Fig. 6(c)). The opening in the mode shape increases as $a_{t}$ increases, which corresponds to the increasing difference between the 'constrained mode' and the 'free mode' frequencies (Fig. 5). For very long delaminations $\left(a_{t} / L>0.8\right)$, local vibration 


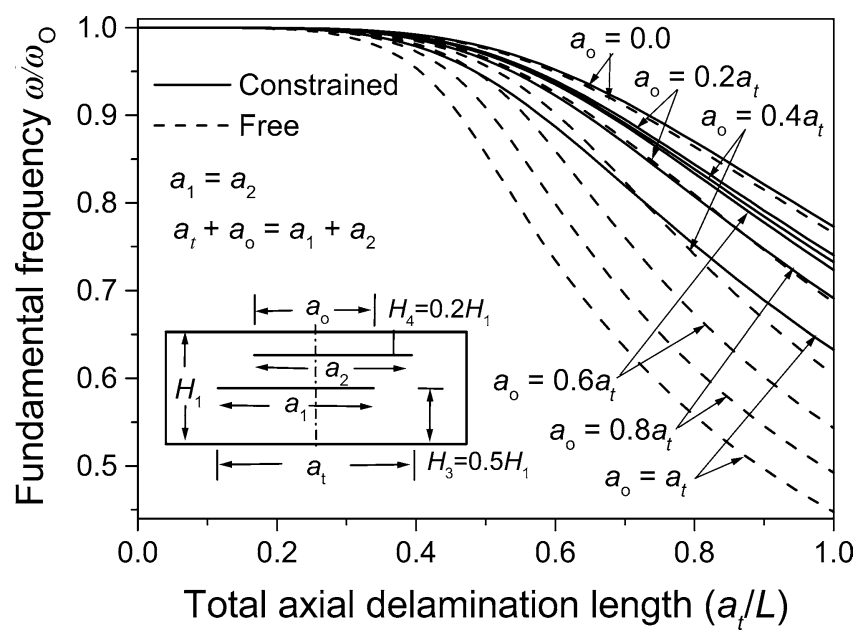

Fig. 5 Influence of the overlapping length, $a_{o}$, and $a_{t}$ on the fundamental frequency of a clamped-clamped beam

modes (e.g. Fig. 6(c)) and relatively low frequencies (Fig. 5) are observed.

Figure 7 shows the influence of the length of the surface delamination, $a_{2}$, on the fundamental frequency of the beam, $\omega / \omega_{o}$. Delamination $a_{1}$ is at midplane $\left(H_{3}=0.5 H_{1}\right)$ and midspan $\left(d_{1} / L=0.0\right)$. The surface delamination is at $H_{4}=0.2 H_{1}$ and spanwisely located at the right end of the midplane delamination $\left(d_{2}=0.5 a_{1}\right)$. For the 'constrained mode', the influence of $a_{2}$ on $\omega / \omega_{o}$ is negligible for very short delaminations $\left(a_{2}<0.2 L\right)$ and remains to be small for up to about $a_{2}=0.3 L$. The influence of $a_{2}$ on $\omega / \omega_{o}$ becomes significant for longer delaminations $\left(a_{2}>0.3 L\right)$. For the 'free mode', the influence of the overlapping delamination becomes significant for $a_{2}>0.2 L$.

The variation of the frequency $\omega / \omega_{o}$ with respect to the surface layer thickness $H_{4}$ is examined in Fig. 8 for various surface delamination length $a_{2}$. The spanwise location of the surface delamination is $d_{2}=0.25 \mathrm{~L}$, while the other delamination with length $a_{1}=0.5 \mathrm{~L}$ is at midplane $\left(H_{3}=0.5 H_{1}\right)$ and midspan $\left(d_{1}=0.0\right)$. For the 'constrained mode', $\omega / \omega_{o}$ decreases as $H_{4}$ increases. This is because the bending stiffness of the beam decreases as the delamination moves towards the midplane [2,3]. However, the decrease is less significant for very short delaminations $\left(a_{2}<0.2 L\right)$. For the 'free mode', $\omega / \omega_{o}$ increases rapidly until it reaches a kink (e.g. (b)). $\omega / \omega_{o}$ decreases as $H_{4}$ further increases up to about $H_{4}=0.35 H_{1}$, after which $\omega / \omega_{o}$ increases slightly and finally decreases.

To investigate the variation of the 'free mode' frequency, the mode shapes for the circled geometries in Fig. 8 are computed and shown in Fig. 9. For a thin surface layer $\left(H_{4}=\right.$ $\left.0.1 H_{1}\right)$, beam 4 dominates the fundamental frequency $\omega / \omega_{o}$. Beam 4 deforms more as compared with the lower beams 5 and 6 (Fig. 9(a)), which results in a local vibration mode and a low frequency. As $\mathrm{H}_{4}$ increases, beam 4 thickens and $\omega / \omega_{o}$ increases. In Fig. 8, the frequency decreases from (b) to $(\mathrm{c})$. Figures $9(\mathrm{~b}, \mathrm{c})$ show a change in the boundary conditions of beam 4 due to the deformations of beams 2 and 5 . The boundary conditions of beam 4 shifts from clamped-clamped
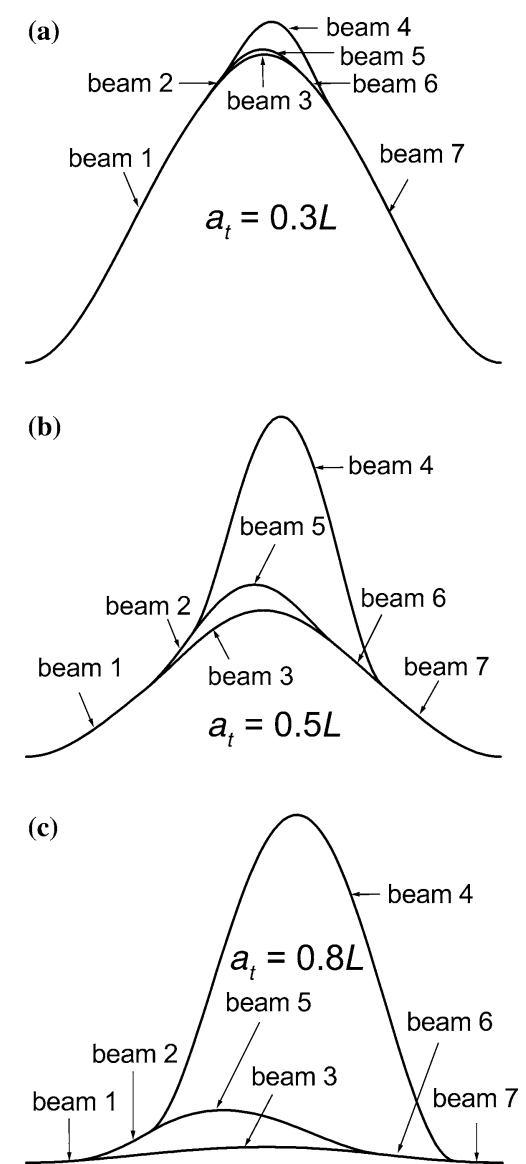

Fig. 6 'Free mode' mode shape of a beam with two overlapping delaminations at $a_{o}=0.6 a_{t}$ and $H_{4}=0.2 H_{1}$ : (a) $a_{t}=0.3 L$; (b) $a_{t}=0.5 \mathrm{~L}$; (c) $a_{t}=0.8 \mathrm{~L}$

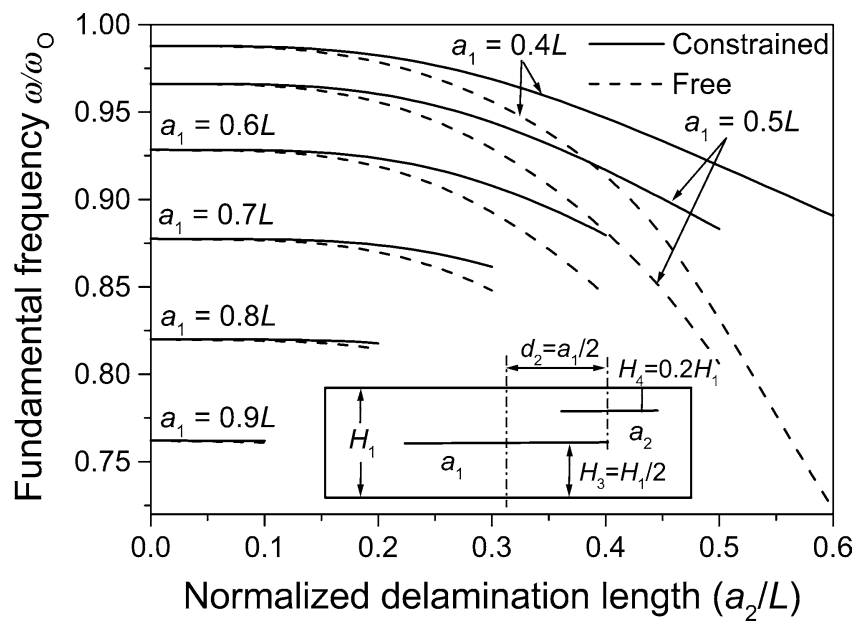

Fig. 7 Influence of the overlapping delamination length, $a_{2}$, on the fundamental frequency of a clamped-clamped beam

$(\lambda=4.73)$ to simply-supported-clamped $(\lambda=3.93)$. This weakens beam 4 and makes $\omega / \omega_{o}$ decrease. $\omega / \omega_{o}$ increases after about $H_{4}=0.35 H_{1}$ (Fig. 8). Figures 9(c,d) show a slight change in the mode shape, which indicates that the increase in $\omega / \omega_{o}$ is due to the thickening of beam 4. Finally, $\omega / \omega_{o}$ decreases (Fig. 8), while thin beam 5 dominates the 


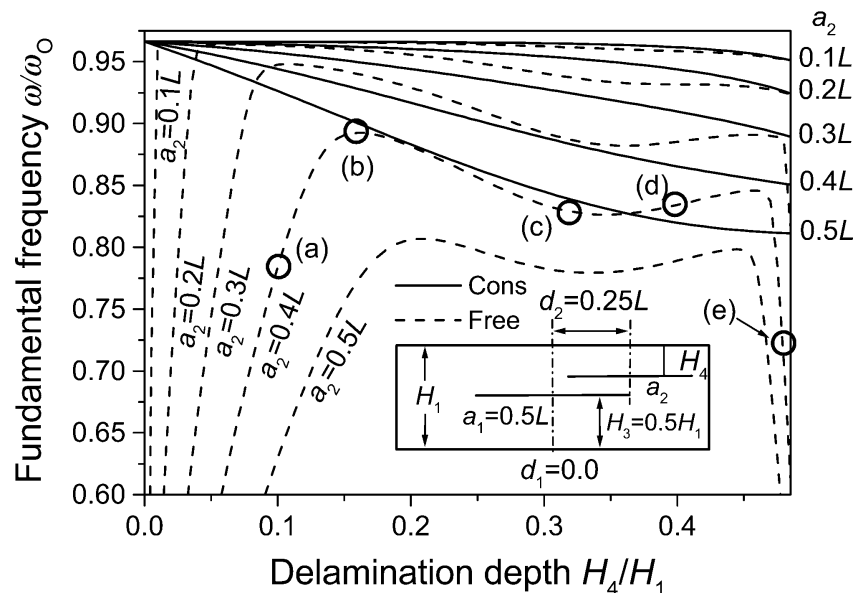

Fig. 8 Variation of the fundamental frequency $\omega / \omega_{0}$ with respect to the surface delamination depth $\mathrm{H}_{4}$

fundamental frequency. Figure 9(e) shows a local vibration mode where beam 5 deforms more than the other beams, resulting in a low frequency. It should be noted that overlapping in the mode shape occurs due to the assumption of the 'free mode' that the delaminated beams deform 'freely' and have different transverse deformations.

Figure 10 shows the influence of the spanwise distance of the surface delamination, $d_{2}$, on the frequency of the beam

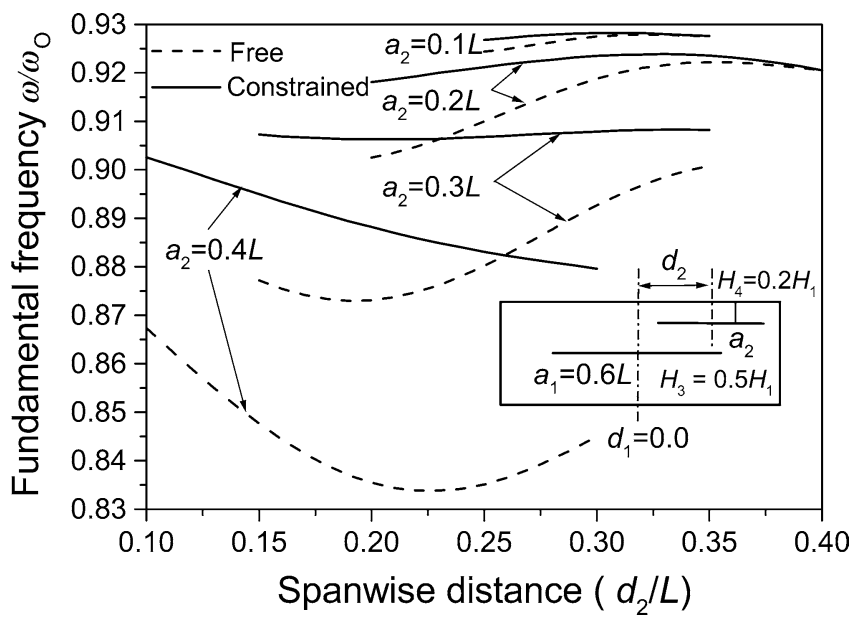

Fig. 10 Fundamental frequency versus the spanwise location of the surface delamination $\left(d_{2} / L\right), H_{4}=0.2 H_{1}, H_{5}=0.3 H_{1}, H_{3}=0.5 H_{1}$

$\omega / \omega_{o}$. The surface delamination is at $H_{4}=0.2 H_{1}$. The length of the midplane and midspan delamination is $a_{1}=0.6 \mathrm{~L}$. The locations of the delamination boundaries satisfy the requirement $x_{6}>x_{5}>x_{4}$ in Fig. 1. The range of $d_{2}$ is therefore limited by $\left(a_{1}-a_{2}\right)<2 d_{2}<\operatorname{Min}\left(L-a_{2}, a_{1}+a_{2}\right)$. For the 'constrained mode', for very short delaminations $\left(a_{2}<0.2 L\right)$, $\omega / \omega_{o}$ increases slightly as the delamination moves towards the beam end. Frequency decreases as the delaminations move
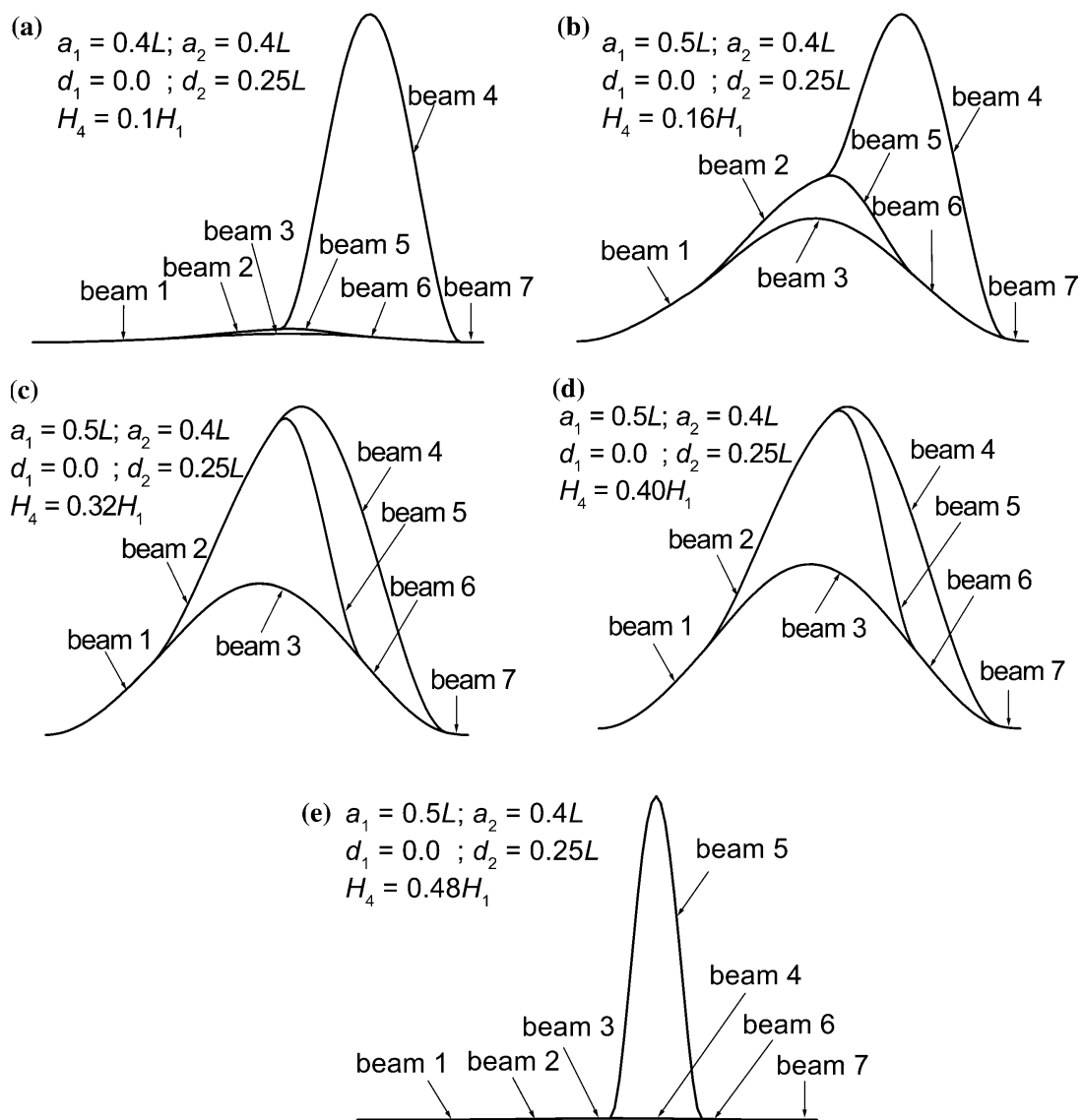

Fig. 9 Vibration modes at various delamination depth for a clamped-clamped beam: (a) $H_{4}=0.1 H_{1} ;\left(\right.$ b) $H_{4}=0.16 H_{1} ;\left(\right.$ c) $H_{4}=0.32 H_{1}$; (d) $H_{4}=0.4 H_{1}$; (e) $H_{4}=0.48 H_{1}$ 
towards the beam end due to the decrease in the differential stretching [16]. However, for very short delaminations $\left(a_{2}<\right.$ $0.2 L)$, the decrease in the differential stretching is negligible, and the increase in $\omega / \omega_{o}$ is due to the increase of the bending stiffness of the beam as the delaminations move towards the beam end. When $a_{2}=0.3 L$, an almost constant $\omega / \omega_{o}$ is observed. This is because the increase in the bending stiffness is balanced by the decrease in the differential stretching as the surface delamination moves towards the beam end. When $a_{2}=0.4 L, \omega / \omega_{o}$ monotonically decreases because of the significant decrease on the differential stretching. For the 'free mode', when $a_{2}<0.3 L, \omega / \omega_{o}$ increases as the delamination moves towards the beam end; on the other hand, when $a_{2}=0.4 L, \omega / \omega_{o}$ increases and then decreases. In addition, for short delaminations $\left(a_{2}<0.3 L\right)$, the largest difference between the 'constrained mode' and the 'free mode' frequencies occurs when the shorter delamination is fully overlapped by the longer delamination. The difference decreases as the delamination moves towards the beam end. This indicates that the mode shapes displays a large opening when the shorter delamination is fully overlapped by the longer delamination and a smaller opening when the shorter delamination is near the beam end.

\section{Conclusions}

The free vibration of composite beams with two overlapping delaminations is solved analytically without resorting to a numerical approximation. The influence of the delamination sizes and locations on the fundamental frequency as well as the mode shapes is investigated. The results of the present solution agree well with the analytical and experimental data reported in the literature. Results show that the sizes and the locations of the delaminations significantly influence the frequency of the beam. However, a short overlapping delamination $\left(a_{2}<0.3 \mathrm{~L}\right)$ does not significantly influence the frequency of the beam. The difference between the 'constrained mode' and 'free mode' frequencies largely depend on the opening in 'free mode' mode shapes. When the mode shape displays a large opening, the difference between two frequencies is large. When the mode shape displays a small opening, the difference between two frequencies is small.

The results are only for a homogeneous beam. When the laminates are of different materials, it is expected that the results will be different quantitatively. However, the trends observed with respect to the depth, the length and the spanwise location of the delamination are expected to be the same.

\section{References}

1. Wang, J.T.S., Liu, Y.Y., Gibby, J.A.: Vibration of split beams. J. Sound Vib. 84(4), 491-502 (1982)
2. Mujumdar, P.M., Suryanarayan, S.: Flexural vibrations of beams with delaminations. J. Sound Vib. 125(3), 441-461 (1988)

3. Shu, D., Fan, H.: Free vibration of a bimaterial split beam. Compos Part B 27(1), 79-84 (1996)

4. Hu, J.S., Hwu, C.: Free vibration of delaminated composite sandwich beams. AIAA J. 33(10), 1911-1918 (1995)

5. Tracy, J.J., Pardoen, G.C.: Effect of delamination on the natural frequencies of composite laminates. J. Compos Mater 23(12), 12001215 (1989)

6. Valoor, M.T., Chandrashekhara, K.: A thick composite-beam model for delamination prediction by using neural networks. Compos Sci Tech 60(9), 1773-1779 (2000)

7. Shen, M.-H.H., Grady, J.E.: Free vibrations of delaminated beams. AIAA J. 30(5), 1361-1370 (1992)

8. Luo, H., Hanagud, S.: Dynamics of delaminated beams. Int. J. Sol Strucs. 37(10), 1501-1519 (2000)

9. Saravanos, D.A., Hopkins, D.A.: Effects of delaminations on the damped characteristics of composite laminates: analytical and experiments. J. Sound Vib. 192(5), 977-993 (1996)

10. Chakraborty, A., Roy Mahapatra, D., Gopalakrishnan, S.: Finite element analysis of free vibration and wave propagation in asymmetric composite beams with structural discontinuities. Compos. Struc. 55(1), 23-36 (2002)

11. Zak, A., Krawczuk, M., Ostachowicz, W.: Numerical and experimental investigation of free vibration of multilayer delaminated composite beams and plates. Comput. Mech. 26(3), 309-315 (2000)

12. Zak, A., Krawczuk, M., Ostachowicz, W.: Vibration of a delaminated composite plate with closing delamination. J. Intell. Mater. Sys. Struc. 12(8), 545-551 (2001)

13. Chattopadhyay, A., Radu, A.G., Dragomir-Daescu, D.: A higher order theory for dynamic stability analysis of delaminated composite plates. Comput. Mech. 26(3), 302-308 (2000)

14. Radu, A.G., Chattopadhyay, A.: Dynamic stability analysis of composite plates including delaminations using a higher order theory and transformation matrix approach. Int. J. Sol. Struc. 39(7), 1949$1965(2002)$

15. Hu, N., Fukunaga, H., Kameyama, M., Aramaki, Y., Chang, F.K. Vibration analysis of delaminated composite beams and plates using higher-order finite element. Int. J. Mech. Sci. 44(7), 1479-1503 (2002)

16. Shu, D.: Vibration of sandwich beams with double delaminations Compos. Sci. Tech. 54(1), 101-109 (1995)

17. Lestari, W., Hanagud, S.: Health monitoring of structures: multiple delamination dynamics in composite beams. In: Proceedings of the 40th AIAA/ASME/ASCE/AHS/ASC Structures, Structural Dynamics and Materials Conference and Adaptive Structures Forum, St. Louis, MO, April 1999

18. Lee, S., Park, T., Voyiadjis, G.Z.: Vibration analysis of multipledelaminated beams. Compos. Part B 34(7), 647-659 (2003)

19. Shu, D., Della, C.N.: Vibrations of multiple delaminated beams Compos. Struc. 64(3-4), 467-477 (2004)

20. Shu, D., Della, C.N.: Free vibration analysis of composite beams with two non-overlapping delaminations. Int. J. Mech. Sci. 46(4), 509-526 (2004)

21. Ju, F., Lee, H.P., Lee, K.H.: Free-vibration analysis of composite beams with multiple delaminations. Compos. Eng. 4(7), 715-730 (1994)

22. Lee, J.: Free vibration analysis of delaminated composite beams. Comput. Struct. 74(2), 121-129 (2000)

23. Ju, F., Lee, H.P., Lee, K.H.: Finite element analysis of free vibration of delaminated composite plates. Compos. Eng. 5(2), 195-209 (1995)

24. Cho, M., Kim, J.-S.: Higher-order zig-zag theory for laminated composites with multiple delaminations. J. Appl. Mech. 68(6), 869-877 (2001)

25. Kim, S.H., Chattopadhyay, A., Ghoshal, A.; Characterization of delamination effect on composite laminates using a new generalized layerwise approach. Comput. Struc. 81(15), 1555-1566 (2003) 
26. Kim, S.H., Chattopadhyay, A., Ghoshal, A.: Dynamic analysis of composite laminates with multiple delaminations using improved layerwise theory. AIAA J. 41(9), 1771-1779 (2003)

27. Shu, D., Mai, Y.-W.: Delamination buckling with bridging. Compos. Sci. Tech. 47(1), 25-33 (1993)
28. Shu, D., Mai, Y.-W.: Buckling of delaminated composites re-examined. Compos. Sci. Tech. 47(1), 35-41 (1993)

29. Shu, D., Mai, Y.-W.: Effect of stitching on interlaminar delamination extension in composite laminates. Compos. Sci. Tech. 49(2), 165-71 (1993) 